Décadrages Décadrages

cinéma, à travers champs Cinéma, à travers champs

$7 \mid 2006$

Stephen Dwoskin

\title{
Ballets d'images et de corps : mouvements rythmés dans les premiers courts métrages de Stephen Dwoskin
}

\section{Laurent Guido}

\section{(2) OpenEdition \\ Journals}

Édition électronique

URL : https://journals.openedition.org/decadrages/443

DOI : $10.4000 /$ decadrages. 443

ISSN : 2297-5977

Éditeur

Association Décadrages

Édition imprimée

Date de publication : 10 avril 2006

Pagination : $42-55$

ISBN : 978-29700582-3-6

ISSN : 2235-7823

Référence électronique

Laurent Guido, «Ballets d'images et de corps : mouvements rythmés dans les premiers courts métrages de Stephen Dwoskin », Décadrages [En ligne], 7 | 2006, mis en ligne le 30 janvier 2014, consulté le 03 avril 2022. URL : http://journals.openedition.org/decadrages/443 ; DOI : https://doi.org/ $10.4000 /$ decadrages.443 


\section{Ballets d'images et de corps: mouvements \\ rythmés dans les premiers courts métrages}

de Stephen Dwoskin

par Laurent Guido

1 Cet article se situe dans le prolongement d'une recherche menée en 2000 , publiée dans Hors-Champ, $\mathrm{n}^{\circ} 4$, printemps 2000, pp. 32-41 (L. Guido, "Bouclées!", "La caméra au rythme des corps").

2 Les modèles sont seules dans Asleep (Sue, la femme du cinéaste), Alone (Zelda), Naissant (Beverly Grant), Moment (Tina Fraser) et Trixi (Beatrice Cordua). Elles forment un couple dans Chinese Checkers (Joan Adler et Beverly Grant), Dirty (Barbara et Ann) et To Tea ("Two T": Tineke et Trix; cette dernière est l'épouse du cinéaste néerlandais Frans Zwartjes, dans la maison duquel a été tourné le film). Voir Stephen Dwoskin, Film Is. The International Free Cinema, Londres, Peter Owen, 1975, p. 210. Quant aux espaces filmés, ils se réduisent à une pièce (Chinese Checkers, Trixi, To Tea) et même à un lit (Asleep, Alone, Naissant, Moment, Dirty).

3 Paul Willemen, "Voyeurism, the Look and Dwoskin", Afterimage, nº, été 1976, pp. 4051. Repris dans P. Willemen, Looks and Frictions, Indiana University Press, Bloomington \& Indianapolis, 1994 [Chapitre 4: "The Fourth Look", pp. 99-110]).

4 Laura Mulvey, "Visual Pleasure and Narrative Cinema", dans The Sexual Subject: A Screen Reader in Sexuality, Londres, Routledge, 1991, pp. 22-33 [1975].

5 Paul Willemen, Looks and Frictions, op. cit., p. 105.
Cette étude porte sur une série de courts métrages tournés par Stephen Dwoskin au début de sa carrière: Asleep (1961), Alone (1963), Chinese Checkers (1963/1965), Naissant (1964), Moment (1968-1969), Trixi (1969), To Tea (1970), et Dirty (1965-1971)1. Ces films s'attachent tous à la même mise en scène de jeunes femmes évoluant, seules ou en couple, dans des décors extrêmement dépouillés 2 . Ils reconduisent donc un même dispositif particulier, fondé sur le rapport noué entre la caméra et le(s) modèle(s). Dans un article célèbre ${ }^{3}$, Paul Willemen a traité plus spécifiquement de la question du regard dans ces premiers films de Dwoskin, qu'il voit contribuer à un dévoilement des mécanismes d'identification visuelle en jeu dans le cinéma dominant. Aux regards successifs de la caméra, du spectateur et des personnages au sein de la diégèse, qui soumettent inlassablement le corps féminin à la pulsion scopique masculine (Laura Mulvey4), s'ajouterait ainsi la possibilité d'un "quatrième regard", celui du sujet filmé vers l'objectif, constituant dès lors une figure d'interpellation susceptible de mettre à nu le système voyeuriste.

Pour appréhender chez Dwoskin cette relation singulière entre le corps et la caméra, Willemen souligne lui-même le caractère essentiel d'aspects esthétiques qu'il ne prend pourtant pas en compte dans son analyse, à savoir «l'importance de la musique, le langage verbal, le jeu sur les éléments structuraux du cinéma» $\mathbf{5}$. Négligée dans les diverses études sur le cinéaste, cette dimension "structurelle» de son travail me paraît en effet tout à fait primordiale. Par sa formation de graphiste au sein de l'underground new-yorkais - il y a débuté en compagnie d'Andy Warhol, Jack Smith, Shirley Clarke ou Jonas Mekas - la démarche de Dwoskin participe à l'évidence des pratiques minimalistes qui marquent le champ artistique des années 1960-1970. Le réalisateur rappelle à cet égard l'inscription de ses premiers travaux dans «la conception américaine des arts 

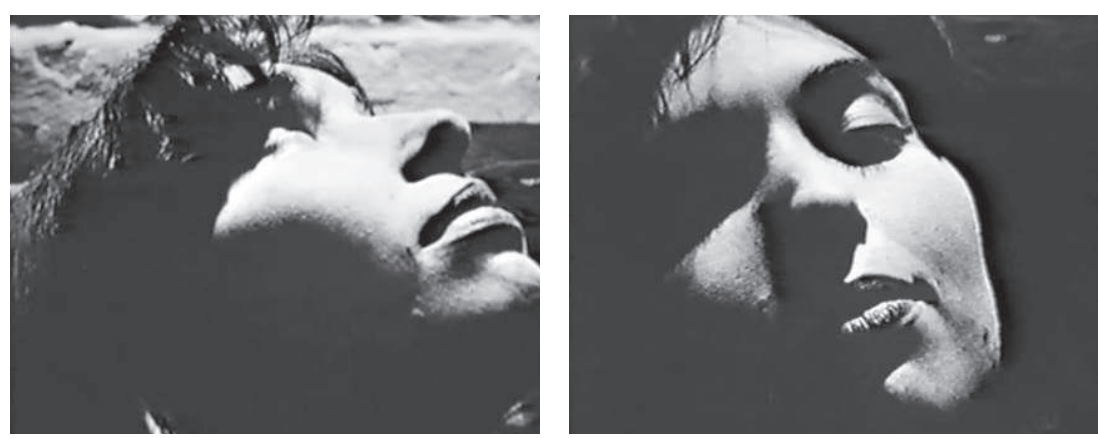

graphiques, c'est-à-dire savoir utiliser tous les médias pour communiquer», en recourant à des "compétences en peinture, en son, en musique et en image photographique». Envisagé comme une nouvelle mouture du Gesamtkunswerk, le cinéma a représenté à ses yeux un mode d'expression privilégié pour les artistes polyvalents de la mouvance underground: "Si on dressait la liste des personnalités importantes de cette époque, on réaliserait qu'en fait toute personne travaillant dans les arts, y compris les danseurs, travaillaient avec le medium cinéma» 6 . Cette référence à la danse témoigne d'une sensibilité particulière envers l'art chorégraphique, qui s'origine notamment dans une expérience familiale. Avant d'être atteint de poliomyélite, Dwoskin a suivi dans sa jeunesse les leçons de son grand-père, professeur de danse en Russie: «en ce sens, le rythme et la musique sont devenus une part innée de mon existence». Nul doute dès lors que la problématique de la musicalité du mouvement filmique figure parmi les préoccupations essentielles du cinéaste. Actualisant la nécessité de développer les potentialités du cinéma à partir d'un modèle musical ou chorégraphique, comme l'avaient prôné les premiers théoriciens du film ${ }^{7}$, les courts métrages de Stephen Dwoskin déploient effectivement des variations systématiques autour de principes rythmiques, dans leurs aspects spatiaux comme temporels, plastiques comme sonores. A l'instar des compositions de Steve Reich, auquel se réfère explicitement Dwoskin 8, ou de John Adams, où l'organisation de l'espace sonore se fonde sur une "progression d'augmentation graduelle ou de prolongation des sons individuels au sein d'un accord répété» 9 , le minimalisme use délibérément de structures itératives, en y introduisant des variations diverses (transposition, intensité, tempo, etc.). Ces procédés engagent, dans les termes de René Passeron, une "esthétique liée à la conception et à la prévision des effets souhaités, plutôt qu'à la pratique, intégrée ou non, d'une répétition instauratrice»10. Cette planification d'effets peut même revêtir le caractère de "trans-

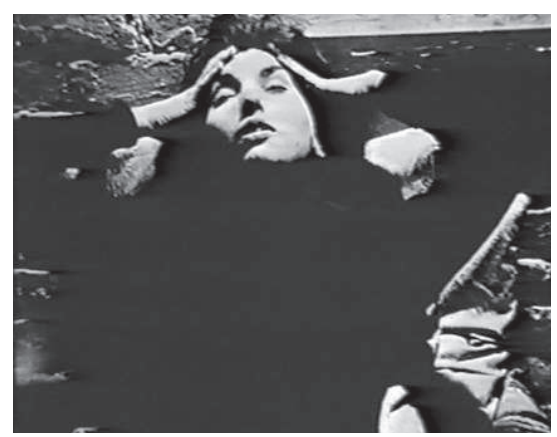

Alone

6 Entretien avec Stephen Dwoskin. Collection privée François Albera.

7 Voir à ce sujet les chapitres 4 et 7 de mon ouvrage L'Age du rythme. Cinéma, musicalité et culture du corps dans les théories françaises des années 1910-1930, Payot, Lausanne, 2006.

8 Dans son ouvrage Film Is (op. cit., p. 218), Stephen Dwoskin rend compte du processus psychologique que cherchent à engager les procédés de composition employés par Reich: susciter le sentiment d'une progression dans la durée, non immédiatement perceptible en raison de son étirement temporel. A cette esthétique minimaliste, il associe directement sa propre démarche, du moins pour son court métrage Moment.

9 Jacques Parrat, Des relations entre la peinture et la musique dans l'art contemporain, Z'Editions, Nice, 1993, p. 34. Voir également Steve Reich, Ecrits et entretiens sur la musique, Christian Bourgois, Paris, 1981.

10 René Passeron, "Poïétique et répétition ", dans Groupe de recherches esthétiques du C.N.R.S., Création et répétition, Editions Clancier-Guénaud, Paris, 1982, p. 15. 
11 Gilles Deleuze, Différence et répétition, P.U.F., Paris, 1969, p. 9 et p. 27.

12 Celle-ci est perceptible dans l'évolution de l'état physique, notamment vestimentaire des personnages. Ainsi le caractère graduel de l'apparition de maquillage sur les visages de Joan et Beverly dans Chinese Checkers ou celui du strip-tease jusqu'à la nudité de Trix dans To Tea et de Beatrice Cordua dans Trixi. Si Dirty est à l'évidence la seule bande non-chronologique du corpus, elle "aboutit " néanmoins à une conclusion du même ordre (l'une des filles baisse le pantalon de l'autre).

13 Illustrations de cette complémentarité symétrique: dans Dirty, deux protagonistes semidévêtues se partagent la même tenue (un pantalon pour l'une; un T-shirt pour l'autre); To Tea oppose Trix, blonde aux longs cheveux frisés, strictement couverte (redingote, jupe longues, bottes hautes) à Tineke, brune à la coupe "garçonne " portant une robe très courte. A la fin du film, la première ne conserve que ses chaussures, le seul accessoire vestimentaire ôté en fin de compte par sa partenaire.

14 Quelquefois nous assistons à une mise en avant progressive de métaphores obscènes: jouer avec une bouteille vide dans Dirty ou se lécher les doigts et s'aider de la main pour engloutir de la nourriture dans To Tea. Le mouvement peut aller jusqu'à une franche exposition du sexe à la caméra (Dirty et Trixi).

15 Ou d'accouchement, comme dans Naissant où les caresses ventrales et les spasmes découlent visiblement de l'enfantement.

$16 \mathrm{~A}$ propos de ce film, Paul Willemen rappelle que le spectateur doit en "construire le iscénario, en combinant une lecture de l'image (légers mouvements de la femme, changements de couleur sur son visage, expressions faciales, etc.) avec une série imaginée (mais suggérée) d'actions situées hors-champ. Le résultat est un récit: une femme qui se masturbe." Steve Dwoskin, A Personal Cinema, Museum of Modern Art, New York, 1978. Programme de la rétrospective, p. 47. gression» que Gilles Deleuze attribue pour sa part à la répétition, dans la mesure où "elle met en question la loi, elle en dénonce le caractère nominal ou général»: "les déguisements et les variantes [...] sont les éléments génétiques internes de la répétition même, ses parties intégrantes et constituantes»11. Au cœur du montage cinématographique, le principe d'alternance implique une telle logique de différenciation/ réitération : fondé sur la reconnaissance et le retour de motifs identiques, le phénomène de la sériation renforce paradoxalement le dynamisme de la continuité filmique, par la constitution progressive d'un continuum à la fois cyclique et vectorisé au fil de transformations et de variations successives. Ce sont ces divers procédés liés à la structuration des films que je souhaite interroger dans les courts métrages de Stephen Dwoskin, tant dans l'agencement du profilmique que dans le cadrage ou le montage.

\section{Leitmotive gestuels}

Si l'interprétation des modèles sur le plan gestuel ne saurait être assimilée à la rigueur d'une chorégraphie ou même d'une partition de rythmique dalcrozienne, elle témoigne généralement d'une attention aiguë du cinéaste pour le mouvement corporel et sa représentation cinématographique. Les activités ludiques ou rituelles montrées dans les courts métrages analysés ici - jouer aux dames chinoises, prendre le thé, s'amuser avec une bouteille, dormir, etc. - constituent un prétexte à la reconduite d'une même trame, aussi minimale soit-elle $\mathbf{1 2}^{\mathbf{2}}$ : après la multiplication de signes de nervosité ou d'impatience, un personnage ou un couple que tout pousse vers la fusion corporelle13 finit par s'adonner aux caresses (intimes ou partagées). Les spasmes ou les convulsions sont alors les signes plus ou moins explicites $\mathbf{1 4}$ d'une libération à valeur d'orgasme ${ }^{\mathbf{1 5}}$, traduite souvent par la seule expression du visage, comme dans Moment où tout se passe hors-champ - avant de décompenser lors des ultimes plans $\mathbf{1 6}$.

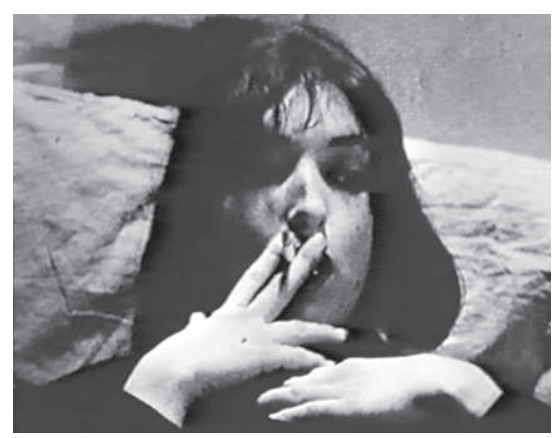

Alone 
Les actions des modèles découlent dans certains films de principes rappelant ceux de la danse. Les Dwoskin girls répètent certaines poses ou attitudes corporelles, presque comme les unités minimales d'un catalogue de petits motifs mimiques. Au fil d'Alone, Zelda adopte effectivement une série de postures, éléments d'une gestique obsessionnelle qui émailleront les autres courts métrages ${ }^{17}$ : elle s'humecte les lèvres en une moue bien marquée, se passe la main dans les cheveux, ne cesse de déplier/replier ses jambes aux frontières du cadre ${ }^{18}$. Si certaines de ces attitudes récurrentes trahissent les signes prosaïques de l'ennui ou du désœuvrement (soupirer; tirer une bouffée de cigarette; se frotter le nez; tapoter de la main; se curer les ongles) ${ }^{19}$, d'autres consistent en des postures très artificielles, comme poser les deux mains jointes sur la poitrine, les croiser sur les épaules ou les placer derrière la nuque 20 . La pose où elle penche la tête en arrière, les yeux fermés $\mathbf{2 1}$, est sans conteste la figure qui sert le mieux les objectifs d'un processus de gradation, dans le sens où elle tend à s'affirmer en violence et en intensité (P21 et 24), ainsi qu'en fréquence (4 fois dans le seul plan 32).

Outre cette dimension mécanique et répétitive, l'aspect chorégraphique peut encore se traduire par des gestes connotant le détachement et l'indolence des protagonistes. Ainsi les actrices de Chinese Checkers finissent par balancer la tête dans une danse lascive. Dans To Tea, Tineke oscille quant à elle avec lenteur et application entre diverses figures : elle tourne sur elle-même; s'appuie sur un pied; prend une posture mélancolique, la tête appuyée contre le mur; souligne un rythme imaginaire par des dodelinements; hausse les épaules de manière récurrente, etc.

\section{L'œil et l'objectif: un pas de deux}

Alternant souvent avec des coups d'œil lancés vers les autres coins du cadre ou des poses avec les yeux clos, les fameux regards vers l'objectif mis en évidence par Paul Willemen peuvent être rapportés à des schèmes

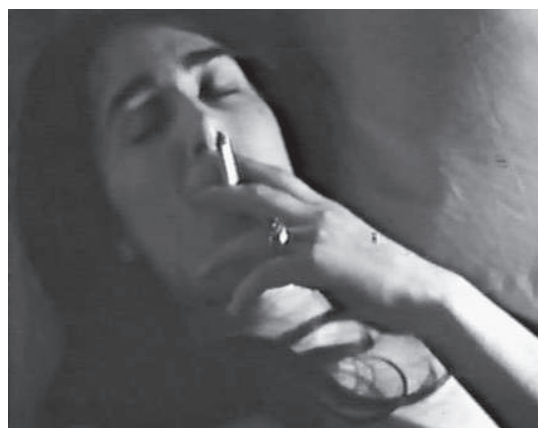

Moment

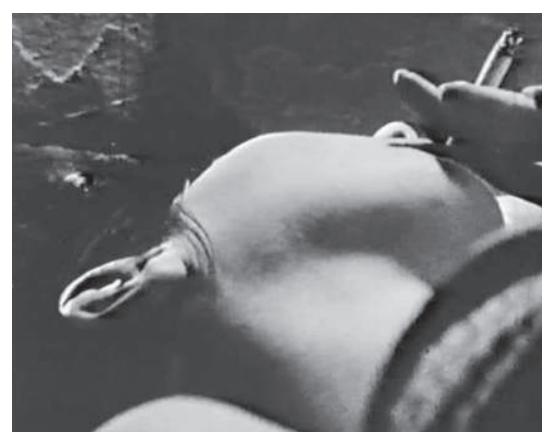

Naissant

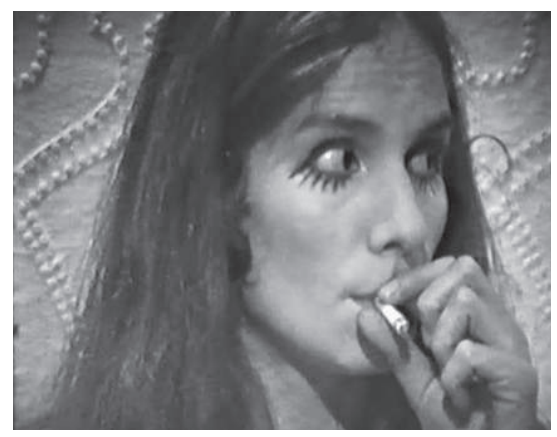

Trixi
17 Moment (9x la bouffée de cigarette, 14x la moue caractéristique), Naissant ( $3 x$ la bouffée, $1 x$ la moue, $6 x$ la tête penchée en arrière, $6 x$ la main passée dans les cheveux, 1x l'inspection des doigts, $3 x$ le soupir, $3 x$ les mains sur la poitrine) et Trixi (6x la moue, $6 x$ la bouffée de cigarette, $18 x$ la main passée dans les cheveux).

$18 \mathrm{P} 2,3,7,10,12,15,16,17,19,24,25,30$, 31, 31 (moue); P14, 15, 16, 17, 18, 19 (mains/ cheveux); P12, 17, 21, 29, 32 (jambes dépliées); P12, 16, 21, 25, 26, 29, 31, 32 (jambes repliées).

$19 \mathrm{P} 4,6,9,10,13,16,17,18,18,19,21,22$ (soupirs); P3, 4, 5, 9, 9 (cigarette); P3, 9, 15 (nez); P13, 21 (main); P2 (ongles).

20 P17, 18, 21, 24 (mains/poitrine); P17, 20, 22, 25 (mains/épaules); P20, 21, 22, 25 (mains/ nuque).

$21 \mathrm{P} 9,10,12,13,14,14,17,18,19,20,20,21$, 22, 22, 24, 24, 25, 29, 31, 32, 32, 32, 32. 
To Tea
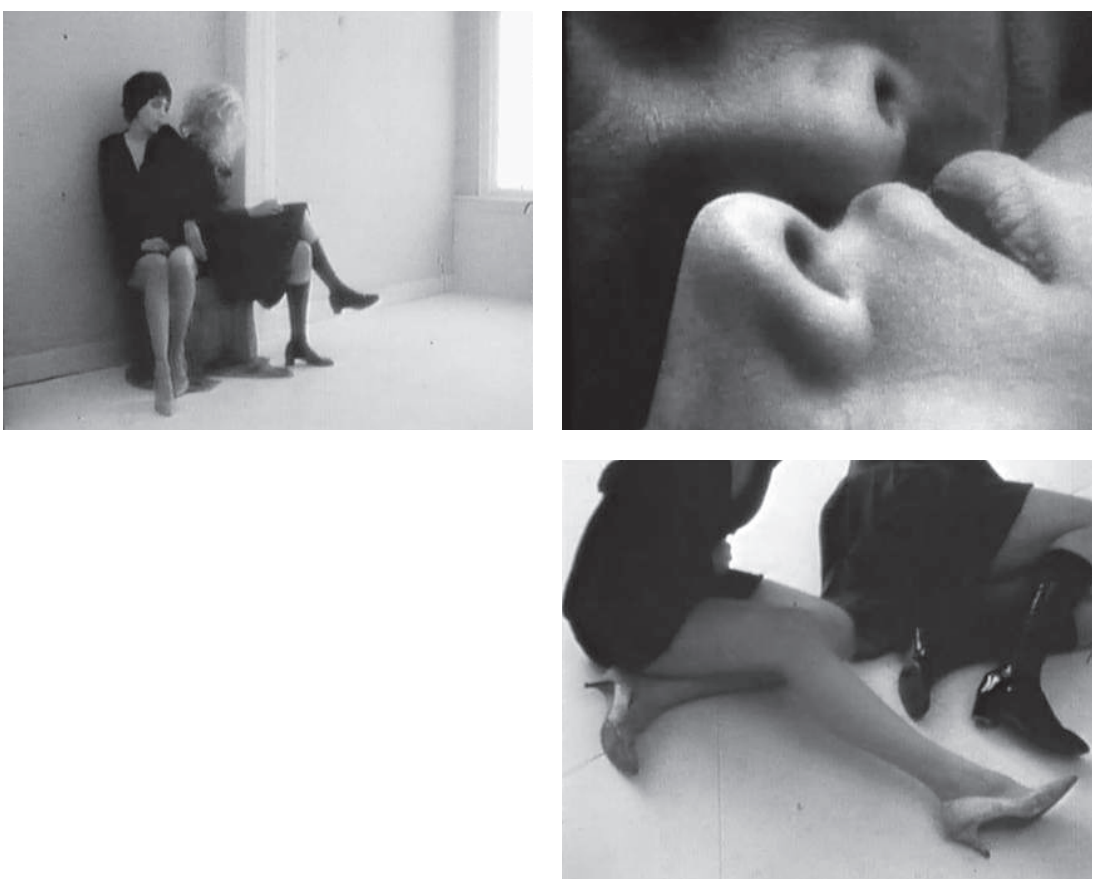

de progression. Ainsi les adresses à la caméra de Moment s'engagent-elles dès le début du film (8 occurrences), alors qu'elles demeurent d'abord indifférenciées dans Alone, à cause du manque de lumière: esquissées aux P2, 7, 8, 10 et 11, elles ne s'affirment effectivement qu'à partir du P12 pour revenir ensuite à 6 reprises et se voir consacrées au P32 par un arrêt sur image final. Plus radicalement encore, c'est dans l'ultime plan de Dirty qu'intervient l'unique regard caméra du film, lancé par l'une des filles. Naissant paraît pour sa part plus marqué par une tension des yeux vers le haut du cadre (15 occurrences marquées), Beverly ne se tournant que deux fois vers la caméra (P9 et 18).

Dans Chinese Checkers, les adresses à la caméra (3 par modèle: Joan P11, 29, 31 ; Beverly P15, 24, 30) se confondent avec les vues subjectives d'un personnage sur l'autre, mimant ainsi un schème de fragmentation "classique». Les deux protagonistes se font face et sont séparées en des champs/contrechamps qui n'obéissent pas vraiment à la règle des $180^{\circ}$ (rupture d'axe au P5). Dans To Tea, la connivence avec la caméra fluctue d'une jeune femme à l'autre: c'est d'abord Tineke qui jette seule des coups d'œil rapides à l'objectif $\mathbf{2 2}$, engageant une relation avec le filmeur/ spectateur qui sera relayée par Trix. Celle-ci commence à dialoguer à son 



tour avec la caméra, à partir du $93^{\mathrm{e}}$ plan. Là, elle se dégage de l'ombre de Tineke qui la masquait et fixe un objectif auquel elle lancera encore trois regards ultérieurs (P125, 126, 127, 166). Ces effets de miroir entre deux personnages qui alternent ou "se passent le regard" disparaissent de Trixi qui procède en quelque sorte à l'affrontement définitif des deux forces de vision en présence, celle du modèle et celle de l'objectif.

Tout au long de Trixi, Beatrice Cordua ne cesse effectivement de se confronter à l'objectif (une soixantaine d'occurrences). Cette interaction s'inscrit dans une certaine progression dramatique. Le regard de la jeune femme est d'abord rapide, exprime la gêne et l'inquiétude avant de se faire interrogateur (P48) et plus soutenu (P49). Il redevient ensuite expression de l'angoisse (P50-52) et du souci (P77), avant de s'apaiser avec la fin du film (associé à un sourire). Avec Trixi, l'acte masturbatoire propre aux films à un seul personnage cède la place à un rapport de fusion entre le sujet filmé et la caméra elle-même.

\section{Chorégraphie de mouvements (d'appareil)}

Dans tous les films analysés, le cinéaste recourt fréquemment aux mouvements de caméra pour faire interagir le rythme du filmage avec celui 
des gestes de modèles. La mobilité de l'appareil peut s'inscrire dans une structure d'ensemble, comme dans Alone où elle se manifeste graduellement après un début exclusivement composé de plans fixes. Dwoskin travaille sur toute l'étendue de la palette des mouvements d'appareil, du tremblement léger suggérant la présence du filmeur au zoom abrupt (Chinese Checkers, To Tea et Trixi). Ce procédé lui permet notamment de se rapprocher vivement des corps filmés. Pour accentuer cet écrasement violent de la distance entre caméra et modèle, le cinéaste prolonge fréquemment le zoom par un effet de flou progressif qui a pour conséquence une déformation des traits, voire leur effacement. Dans Trixi, la caméra scrute ainsi sans relâche le visage de Beatrice Cordua, dévoilant les détails de sa peau en très gros plan (boutons, rides, etc). Les mouvements de caméra y sont frénétiques et continuels, les zooms avant sur le visage omniprésents. A partir du $50^{\mathrm{e}}$ plan, le rapport de la caméra au modèle prend un tour plus dynamique, jouant du croisement des différents paramètres possibles:

P50 [13'38"] A partir d'un plan rapproché du modèle, ample zoom arrière qui reprend le plan introductif (P1). Premier zoom avant en sa direction, qui se stoppe net en plan américain. Elle baisse aussitôt les bras, comme en réaction au déplacement de la caméra. Deuxième zoom avant jusqu'à un plan rapproché. Troisième zoom avant jusqu'à un gros plan du visage. La caméra bouge alors de gauche à droite, en de petits mouvements répétés et rapides. Puis avance progressivement vers le visage jusqu'au flou et au noir le plus complet.

P51 [14'16"] Gros plan fixe (léger tremblement) et net de Beatrice qui regarde vers l'objectif.

P52 [14'41"] Plan rapproché. La caméra avance vers le visage de la fille, jusqu'au flou. L'image devient nette: gros plan du visage aux yeux écarquillés, la bouche ouverte, fixant la caméra. Beatrice glisse alors le long du mur, suivie par la caméra qui s'arrête sur ses seins, alors qu'elle se tourne de côté. La caméra zoome en arrière et reprend le plan introductif (P1). La fille est tournée légèrement de côté. Les cheveux recouvrent son visage. Elle pose alternativement ses mains sur son sexe et ses seins. Elle s'arrête en fixant la caméra, les mains posées le long du corps. Alors qu'elle plonge sa tête en avant, la caméra fait un abrupt zoom avant, jusqu'au bas du visage.

P53 [15'36"] Gros plan frontal du visage. La caméra bouge alors de gauche à droite, en de petits mouvements répétés et rapides. Sourire de la fille qui regarde vers la caméra. 



P54 [15'42"] Idem, elle se baisse, suivie par la caméra. Elle pose sa tête par terre, comme si elle réagissait à l'«assaut» de mini-zooms avant-arrière. Elle se relève brutalement, suivie par la caméra.

P55 [15'55"] Plan rapproché taille. Elle cache son visage de ses deux mains. Zoom avant rapide sur elle. Beatrice se baisse, ne quittant pas la caméra des yeux. Elle est aussitôt suivie par la caméra, en gros plan.

Fondée dans Trixi sur un jeu de rapprochements et d'esquives successifs, cette articulation complexe entre déplacements corporels et cadrage s'accomplit pleinement dans To Tea, qui explore rythmiquement les mouvements d'appareils en fonction de principes de progression. Ainsi les panoramiques ascendants répondent dans ce film à une logique qui vise à découvrir graduellement les corps, dans des plans qui se répondent et se complètent à des étapes différentes de l'œuvre: une même vue sur Tineke debout remonte des jambes à la taille (P8, repris au $\mathrm{P} 16)$, à la poitrine (P14), à la nuque (P15), avant de dépasser finalement la jeune femme pour atteindre le plafond (P55). Dans le même ordre d'idées, la caméra tente de circonscrire le cadre spatial limité dans lequel les modèles se déplacent, soulignant leurs relations internes, toujours dans la verticalité. Elle corrèle par exemple leurs jambes à leurs visages (P27), 
23 Plus loin, toujours dans l'axe vertical, on se déplace encore d'une chaussure disposée sur le plateau à thé jusqu'au plafond, prenant les filles au passage (P97). A ce plan répond clairement un dernier panoramique vertical partant de la fenêtre et aboutissant au soulier initial (P99).

24 Entretien avec Stephen Dwoskin. Collection privée (François Albera).

25 Si Moment consiste en un long plan unique et fixe d'un visage, le corps du modèle d'Asleep se réduit pour sa part au seul détail de ses pieds sortant d'une couverture, comme ceux d'une personne qui dort. Même dans les films marqués par une plus grande diversité de plans, la présentation des modèles s'effectue via des cadres introductifs (P2 d'Alone, plan moyen servant de cadre maximal au film et P4 de Naissant, bande dont le cadrage n'excède jamais le bord de la couverture où est glissée la protagoniste). Enfin, dans Trixi, Beatrice Cordua est d'emblée présentée en plan moyen, assise sur une chaise devant un mur (P1). Dès lors, l'échelle variera entre le très gros plan et le plan américain.

26 Dans Chinese Checkers, le plan initial du damier (P1, qui sera repris aux $P 4,6,8,12,19)$ est suivi par une image des deux modèles assises de profil. Dans les termes propres au découpage hollywoodien, ce plan constitue une sorte de master shot (repris aux P10 et 25) pour la série subséquente de champs/contrechamps entre des gros plans isolés de Joan Adler (P3, 9, 11, 14, 16, 18, 21, 23, 26, 31) et Beverly Grant (P5, 7, 13, 15, 17, 20, 22, 24, 27, 32). La rencontre dans le plan des deux jeunes femmes s'opère par le biais de mouvements de caméra passant par le jeu de dames (P2830). Dès le plan 33, elles sont coprésentes à l'image jusqu'à la fin du film (P38).

27 Le début de To Tea repose également sur une fragmentation plutôt conventionnelle de l'espace. Le premier plan introductif, moyen frontal, est une sorte d'establishing shot: à gauche, Trix est assise, les jambes croisées, les mains posées devant elle. A droite, son corps servant de référence au cadre, se tient Tineke, debout, les mains sur les hanches, sa silhouette se détachant devant la fenêtre. A cette image répondent deux gros plans en raccord dans l'axe avant sur Trix (P2) et Tineke (P3). Dès lors vont se succéder durant le film trois types de plans: sur l'une et l'autre des filles et sur le couple réuni. Les deux "héroïnes" sont fréquemment reliées par des mouvements leurs silhouettes au plafond (P57), le sol filmé en plongée au plafond en passant par elles (P60), puis, provoquant un effet de rétrécissement, du sol aux filles seulement (P63) 23. D’autres mouvements de caméra, horizontaux cette fois, saisissent au passage les filles dans le cadre, le plus souvent en passant d'un mur blanc à un autre: P48, 98, 140, 142, 144, 146-148 (3 plans successifs assemblés avec des jump cuts).

\section{Scansions du montage}

Le même dispositif scénique se retrouve dans l'ensemble des films analysés: un proscenium bien circonscrit, où les modèles évoluent frontalement. Cet agencement découle tout d'abord des conditions particulières de tournage, les mouvements du filmeur étant restreints par son handicap: «Tous les films précédant Bebindert sont basés sur ma personne dans une position de sécurité, tout à fait comme la caméra sur un trépied regardant et observant. [...] C'est cette intensité dans la façon de dévisager, d'étudier et de se servir de ses yeux comme principal facteur de communication, issue du fait d'être handicapé et pas très mobile, qui crée une attitude particulière dans mes films.» 24

En outre, ce dispositif fait écho à la volonté d'installer un système de représentation conventionnel (présentation «classique» des protagonistes, puis stricte logique d'alternance) pour mieux le mettre en crise. Ainsi les bandes consacrées à un seul modèle se caractérisent-elles par la succession de prises de vue rapprochées qui constituent autant de coupes, dans le même axe, sur les corps cadrés par l'objectif 25 . Quant aux films à deux protagonistes, si Chinese Checkers ${ }^{26}$ et To Tea ${ }^{27}$ recon-




duisent avec une certaine rigueur une forme de découpage canonique, la logique d'alternance s'approfondit d'une manière plus novatrice dans Dirty. Dwoskin y analyse puis recompose les actions représentées, à la fois dans leur déroulement interne - fragmentation du plan, segmentation de l'action - et dans l'agencement de différentes séries $\mathbf{2 8}$. Tout comme dans Asleep, où l'usage du pixillating 29 et de l'omission de certains photogrammes rend impossible toute segmentation en "plans", Dirty témoigne d'un travail de manipulation du défilement de la pellicule par le biais de véritables stratégies de déconstruction et de recomposition du mouvement. Cette œuvre résulte en effet du re-filmage de plans tournés six ans auparavant et projetés sur un écran. Le procédé permet non seulement d'extraire des détails ou de circuler à l'intérieur des cadres, qu'ils soient arrêtés ou en mouvement, mais également de jouer des modifications de vitesse: plans statiques; passage de la fixité à l'animation; animation par étapes, c'est-à-dire décomposition du mouvement par arrêts successifs; ralenti, etc. Avec Dirty, Dwoskin expose par ailleurs la matérialité même du film en affichant la dégradation de la pellicule (rayures, déchirures ou poussières), ainsi que les modalités de la projection elle-même (décrochement au P127, reframing de l'opérateur aux P122 et 162) ${ }^{30}$.

La mesure précise des durées de chaque plan permet de dégager certains principes structurels $\mathbf{3 1}$. Les moyennes de certains films se révèlent assez lentes, supérieures à 15 secondes : Naissant (12'45", 34 plans, 21,4 sec./ plan), Chinese Checkers (12'41", 40 plans, 18,5 sec./plan) ou Trixi (26', 95 plans, $15,5 \mathrm{sec} . / \mathrm{plan})$. Si l'on compare ces moyennes avec les durées

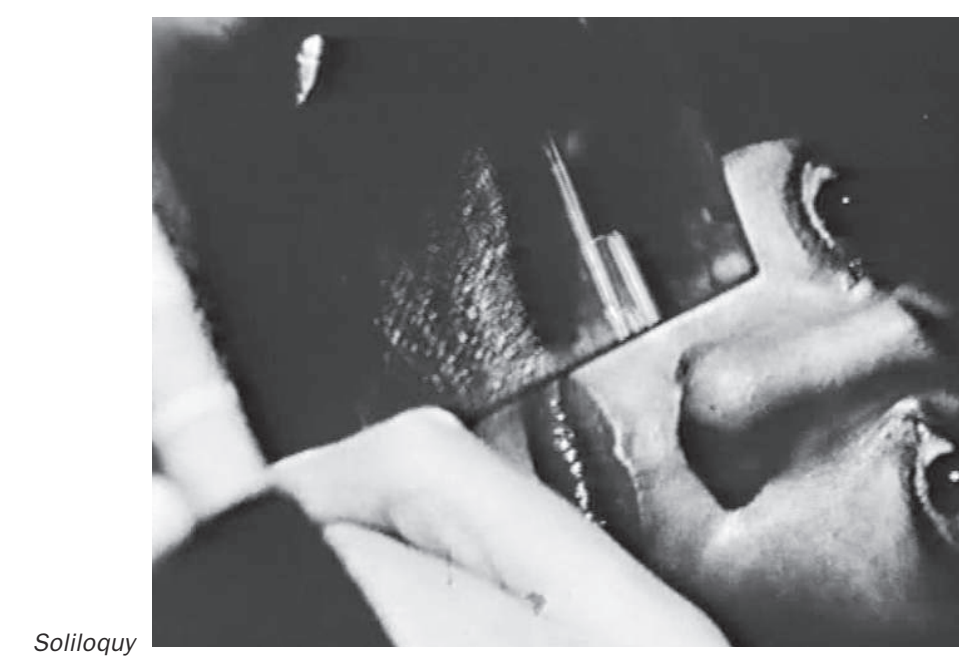

de caméra, images qui prennent souvent des accents fétichistes: gros plans de jambes, de mains, de poitrines, etc., avec une prédilection marquée pour certains leitmotive visuels (escarpins de Tineke, seins découverts de Trix).

28 Par exemple, dans le plan de base A, la caméra part du lit, suit un genou qui s'élève, remonte en panoramique vers une bouteille. Celleci est alors saisie par une main qui pénètre dans le champ. Dans le film, cette action est découpée et présentée dans un ordre non chronologique: d'abord, la bouteille et la main s'en approchant (P1), puis une série de prises reprenant toujours plus bas le panoramique montant du lit à la bouteille (P2, 6, 7). Ce n'est qu'au plan 8 que l'action rattrape le mouvement laissé au P1 (bouteille saisie par la main). Par ailleurs, le film répète certains plans de la série, déjà présentés: $P 1=P 25, P 2=P 4, P 6=P 29, P 7=P 31$ et $P 8=P 26,32,54$. Les mêmes procédés se retrouvent dans les autres séries: B, C, D, E, F et $G$. Les positions de caméra y sont soit frontales ( $D$ et $F$ ), soit décalées sur la gauche (B, $\mathrm{E}$ et $\mathrm{G})$. Les cadres deviennent difficiles à identifier lorsque Dwoskin commence à insérer des plans qui se révèlent être des recadrages à l'intérieur des plans de base, générant notamment deux nouvelles sous-séries: H ("détails " de G) et I ("détails" de F).

29 Enregistrement image par image des actions filmées, comme dans un dessin animé. S. Dwoskin, op. cit., p. 194.

\section{0 ld., p. 181.}

31 Les durées moyennes de plans ont été calculées à partir de celles de chaque plan iconique des films, à l'exception des cartons de générique. 
réelles des plans, on peut les considérer comme plutôt représentatives. S'apparentant selon Dwoskin lui-même à un "flux cohérent donnant l'impression d'un énoncé visuel non-monté et continu »32, Alone (12'49", 32 plans, 22,6 sec/plan) déploie ainsi un mouvement lent et isochrone, s'appuyant par exemple sur une longue série continue de 11 plans (16 à 20) à la durée inlassablement égale (26 ou 27 secondes).

Si les films cités ci-dessus ne manifestent pas des variations de vitesse trop significatives, à l'exception de quelques passages-clé, comme les P50 ou 52 de Trixi analysés plus hauts, qui s'étirent jusqu'à des durées respectives de 48 et 55 secondes. D'autres œuvres, comme Dirty et To Tea, se caractérisent par des moyennes beaucoup plus basses et manifestent une structure "métrique» nettement plus marquée. Regroupées par séries de dix plans, la moyenne de To Tea (21', 192 plans, 6'31"/plan) fait apparaitre un allongement progressif de la durée des cadres (dès le plan $31: 5,2$; $5,2 ; 5,9 ; 5,5 ; 10,1 ; 15,3 ; 17,3)$, un décroissement de la fréquence des changements de plans qui accompagne clairement l'enlacement graduel des deux protagonistes. Appliquée à Dirty (11'25", 162 plans, 3,98 sec./ plan), la même opération dévoile un étirement des plans vers la fin du film (à partir du plan $111: 4,6 ; 4,6 ; 1,6 ; 4,8 ; 10,1 ; 10$ ). Cette œuvre comporte d'ailleurs des passages d'accélération, comme aux plans 42 à 47 (6 plans en 3 secondes) ou 134-142 (9 plans en 7 secondes). Pour les plans 131-141, la moyenne du débit avec lequel se succèdent les images atteint alors le pic de 1,6 seconde. A partir de telles variations de vitesse, qui autorisent la construction d'effets de tension/résolution, Dwoskin suit une démarche récurrente chez les cinéastes expérimentaux attachés à la représentation des mouvements chorégraphiques, de Maya Deren à Anna Teresa de Keersmaker, qui ont puisé leur inspiration dans des formes musicales canoniques, en particulier la structure caractéristique du climax. Celle-ci répond idéalement au micro-drame qui se joue dans les courts métrages de Dwoskin, structurés autour d'une quête lancinante de la fusion corporelle et de l'orgasme.

Pour déceler de telles variations rythmiques dans Moment, long plan continu de 12', il faut nécessairement s'intéresser aux développements inscrits dans le profilmique. Par contre, l'étude précise d'un autre court métrage de Dwoskin, Jesus Blood Never Failed Me Yet (1972) (29'), montre que ce fameux plan unique d'un trottoir londonien emprunté par un vieillard est en réalité constitué d'au moins huit coupes successives, enchaînées par des fondus presque imperceptibles. Le moment où intervient la première ( $\left.2^{\prime} 29^{\prime \prime}\right)$ correspond à une durée bien déterminée. Dès $10^{\prime} 40^{\prime \prime}$, de telles coupes seront effectivement espacées en fonction d'une scansion isochrone : 2'27", 2'31", 2'30", 2'31", 2'30", 2'28" et 2'33"! Ce pro- 

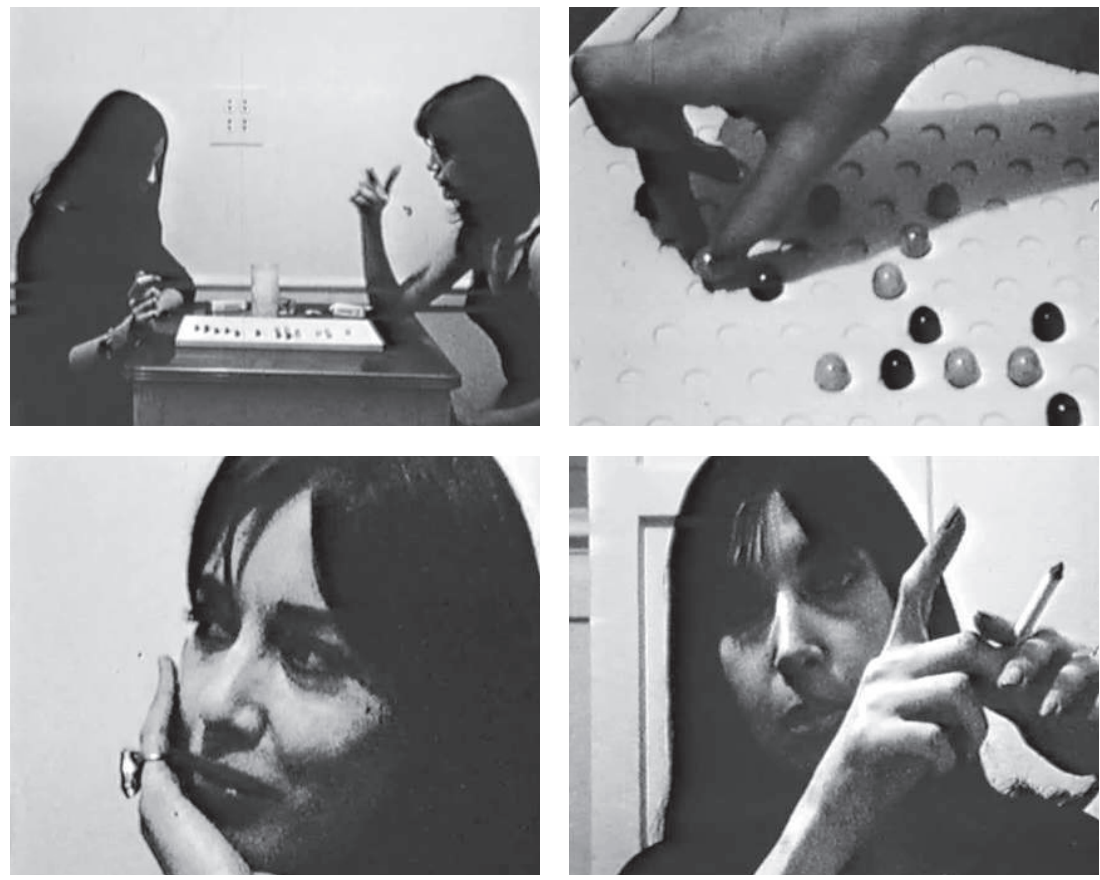

Chinese Checkers

cédé accentue le ralentissement déjà imprimé à la démarche de l'homme via l'emploi d'une caméra spéciale 33 .

\section{Formes musicales}

La musicalité des différents paramètres visuels s'accorde par ailleurs à la musique, instrumentale ou concrète, qui imprègne les films de Dwoskin. Qu'il s'agisse du sound designer Ron Geesin (Asleep, Alone, Chinese Checkers) ou du compositeur minimaliste Gavin Bryars (Naissant, Jesus Blood, Dirty ${ }^{34}$ et To Tea), les sonorités des courts métrages dwoskiniens renvoient aux mêmes processus de répétition minimalistes qui innervent le travail sur les images.

Seule exception à cette influence, l'accompagnement d'Asleep rend un hommage ironique au mickeymousing des dessins animés, coïncidant quelquefois avec des mouvements effectués par les pieds de Sue. Accentuant le ton comique de ce véritable ballet pour orteils, une basse fondée sur une pompe en tierce majeure succède dès le carton MUSIC à une introduction sibylline (accords dissonants, piano désaccordé...). Une mélodie sautillante s'engage sitôt le générique terminé, de surcroît passée à l'accéléré pour souligner le débit frénétique des images. D’autres
33 "L'image de Jesus Blood semble immobile. Pourtant l'homme s'avance bel et bien vers nous, avec une extrême lenteur. Ce film fut tourné avec une caméra médicale à une prise de vue très rapide et une pellicule très sensible." Catalogue du $6^{\text {e }}$ Festival de Marseille, op. cit., p. 47.

34 Je n'ai pu consulter qu'une copie muette du film, en dépit de la mention du travail de Gavin Bryars au générique. 
films insèrent plus subtilement de tels effets d'underscoring comique. Au cours de Chinese Checkers, le démarrage de la basse synthétique coïncide ainsi avec la réapparition d'un master shot (P25) et le début de l'ostinato final rapide souligne exactement la pose d'un collier (P33). Dans Naissant, un pizzicato paraît provoquer soudainement un froncement de sourcil (P10), des effets sonores semblent déclencher un rire (P19) ou un mouvement du bras (P20). Mais les aspects musicaux essentiels et marquants de ces deux bandes se situent véritablement dans leur structure cyclique, leur recours à la répétition et la déformation sonore, autant de traits définitoires de l'univers musical des courts métrages de Dwoskin.

Sur le plan musical, Chinese Checkers glisse ainsi d'une introduction très "espacée» (une note de basse, un silence, une courte réponse mélodique sur une gamme aux consonances asiatiques, interprétée sur un instrument à cordes) à l'élaboration graduelle d'un rythme hypnotique. Dès 4'20", le martèlement de la basse se fait plus insistant, jusqu'à un premier point d'orgue (5'44"). Un motif lent et répétitif en $4 / 4$ se met alors en place à partir d'un son grave déformé électriquement, doublé vers 7'53" par une sorte de basse synthétique. S'y adjoint une voix mélodique aux cordes. De plus en plus free, celle-ci aboutit à la répétition rapide d'une même note, une pulsation qui décélère vers 11'40" pour se terminer en une parodie de conclusion tonale (arpèges d'un accord majeur).

La partie sonore de Naissant débute par une forme d'improvisation libre, voire désinvolte (notes à la justesse approximative, effets de portamento, répétitions rythmiques un peu naïves): une suite de longues notes graves au violoncelle gravitent autour d'un intervalle de quinte diminuée (mi-sib). Dès 3'42", un pizzicato inaugure l'élaboration d'une walking bass lente et monotone (sol-fa-sol-fa dièse), à laquelle se superpose une minute plus tard une mélodie au violoncelle. Vers 6'30", ce solo laisse la place à un effet sonore dans les aigus, dont le volume change brusquement d'intensité. A trois reprises ( $7^{\prime} 42^{\prime \prime}$ et 9' $52^{\prime \prime}$ et $\left.11^{\prime} 45^{\prime \prime}\right)$, le groove en sol se manifeste temporairement, toujours accompagné du solo de violoncelle qui se fait de plus en plus agressif (cordes frappées, grincements à l'archet). La fin du film voit la reprise du motif initial en mi, réitéré dans les graves. Sur l'ultime image, des bruits aigus évoquent une sirène ou le vrombissement de voitures, avant d'être interrompus par un dernier arpège majeur.

La radicalité de la bande son d'Alone (une boucle de 4 secondes, motif de basse en fa répété au piano sur toute la durée du film) et de Moment (un souffle continu et immuable rappelant un bruit de jet d'eau, réverbéré et retravaillé comme un battement percussif) participe de ce même minimalisme. 
Jesus Blood Never Failed Me Yet fournit l'occasion à Dwoskin d'élaborer une œuvre entière à partir d'une pièce musicale, c'est-à-dire le morceau homonyme signé Gavin Bryars 35. Basée sur l'enregistrement de la voix d'un vieillard disparu, tiré d'un documentaire d'Alan Power sur les clochards londoniens, la boucle d'un air traditionnel $\mathbf{3 6}$ est répétée 70 fois, augmentant peu à peu en intensité sonore et bénéficiant d'un accompagnement instrumental qui s'étoffe progressivement avec basse, accords ou arpèges: orchestre de cordes sur la $10^{\mathrm{e}}$ occurrence, guitare ou harpe à la $18^{\mathrm{e}}$, etc. Ce travail sur le mixage, sorte de sampling avant l'heure, trouve son accomplissement dans Trixi, dont la bande son se révèle des plus dépouillées. Répétant sans relâche les deux syllabes «Tri-xi", l'enregistrement d'une voix s'accélère au cours du film, jusqu'à devenir difficilement perceptible: le motif évolue tout d'abord (5' environ) à un taux de 36 occurrences/minute, avant de passer à 38 oc./minute (6 $6^{\mathrm{e}}$ minute), $40\left(11^{\mathrm{e}}\right), 43\left(14^{\mathrm{e}}\right), 46\left(18^{\mathrm{e}}\right), 48\left(20^{\mathrm{e}}\right), 50\left(23^{\mathrm{e}}\right)$ et $52\left(25^{\mathrm{e}}\right)$. L'effet résulte en premier lieu d'un rétrécissement de l'écart entre les occurrences du motif, puis entre les syllabes, et enfin à l'intérieur des syllabes elles-mêmes. La contraction sonore provoque une transformation de la prononciation, permettant à des sons annexes de prendre une nouvelle prépondérance. Ainsi le claquement de la résonance électrique finit-elle par s'apparenter à une sorte de rythme secondaire, fonctionnant comme un écho au motif principal.

Les premiers courts métrages de Stephen Dwoskin témoignent en fin de compte d'une inscription, propre au champ cinématographique underground de son époque, de pratiques émergeant alors dans les domaines de la musique ou des arts plastiques (répétition, minimalisme). Tout en ne répondant pas aux principes très rigoureux déployés par exemple chez Peter Kubelka ou Werner Nekes, cette dimension structurelle mérite d'être pleinement reconnue, dans la mesure où elle ouvre la voie à une exploration des multiples potentialités rythmiques et plastiques du médium cinématographique, tant dans l'interprétation, l'agencement du profilmique, le cadrage, le montage, le découpage de l'espace ou encore les mouvements d'appareils et les relations entre l'image et le son.
35 Jesus Blood Never Failed Me Yet est disponible en CD, chez Point Music (1993), dans une version récente (la plus longue). Cette pièce a été remaniée à plusieurs reprises par Gavin Bryars.

36 "Jesus Blood Never Failed Me Yet never failed me yet / Never failed me yet / Jesus Blood Never Failed Me Yet never failed me yet / There's one thing I know / For He loves me so". 
De 1982 à 1989, Véronique Goël vit et travaille à Londres, avec Stephen Dwoskin. Elle a collaboré au tournage et au montage de Shadows from Light (1983), Ballet Black (1986) et Further and Particular (1988). 

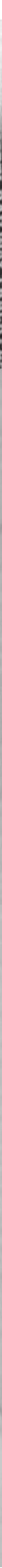

\section{love}
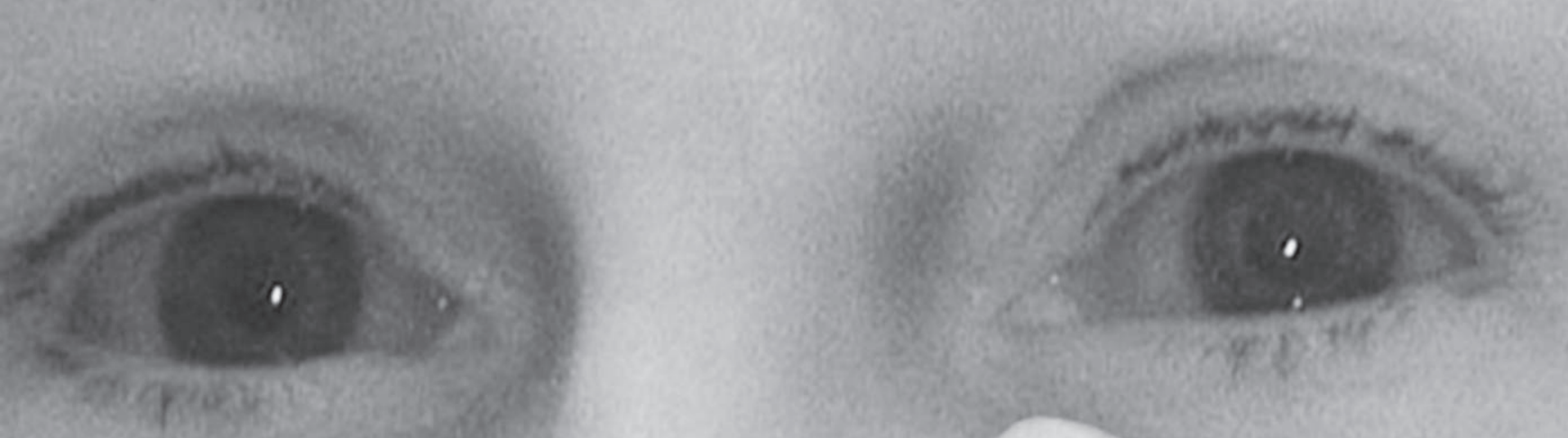


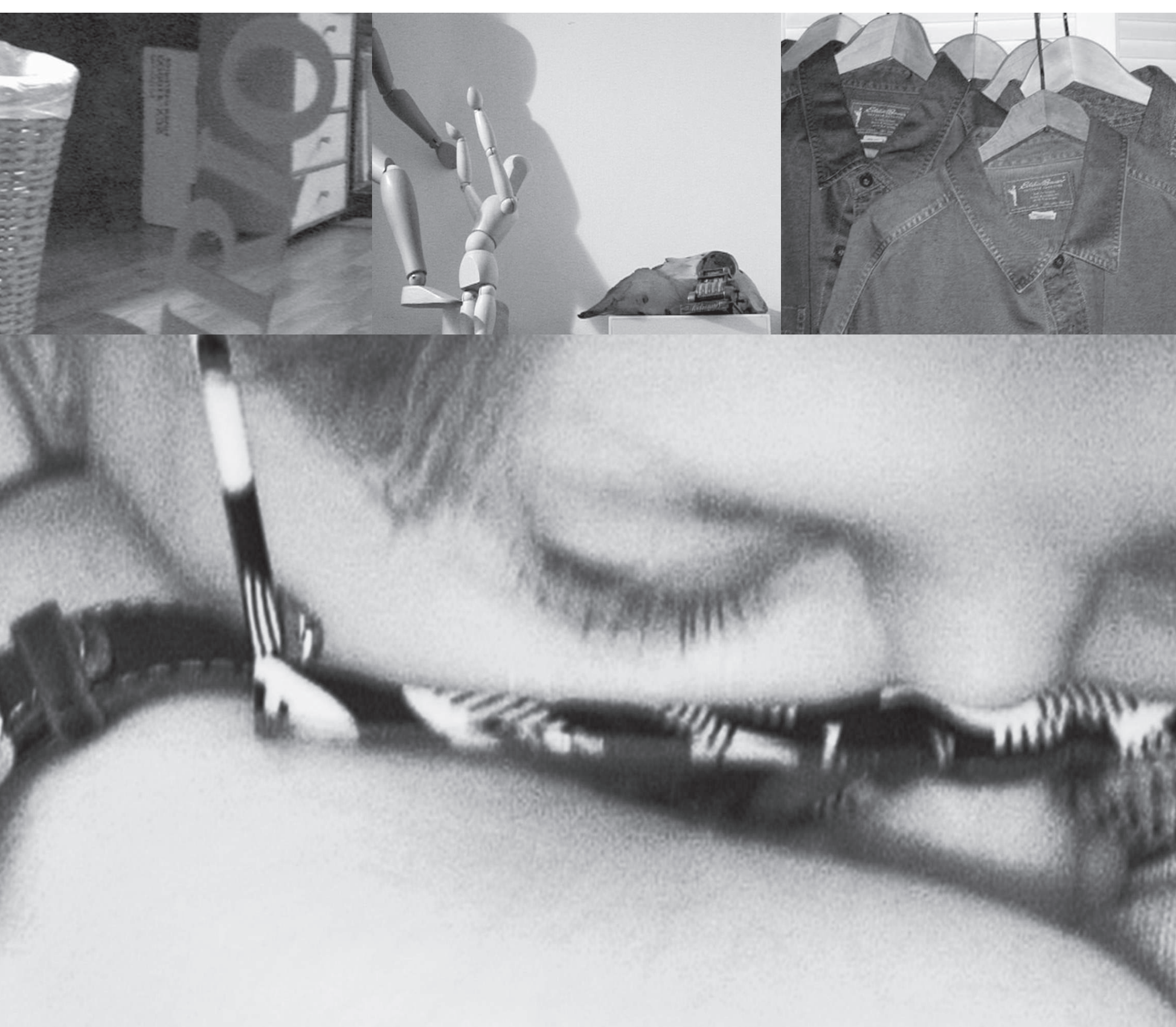




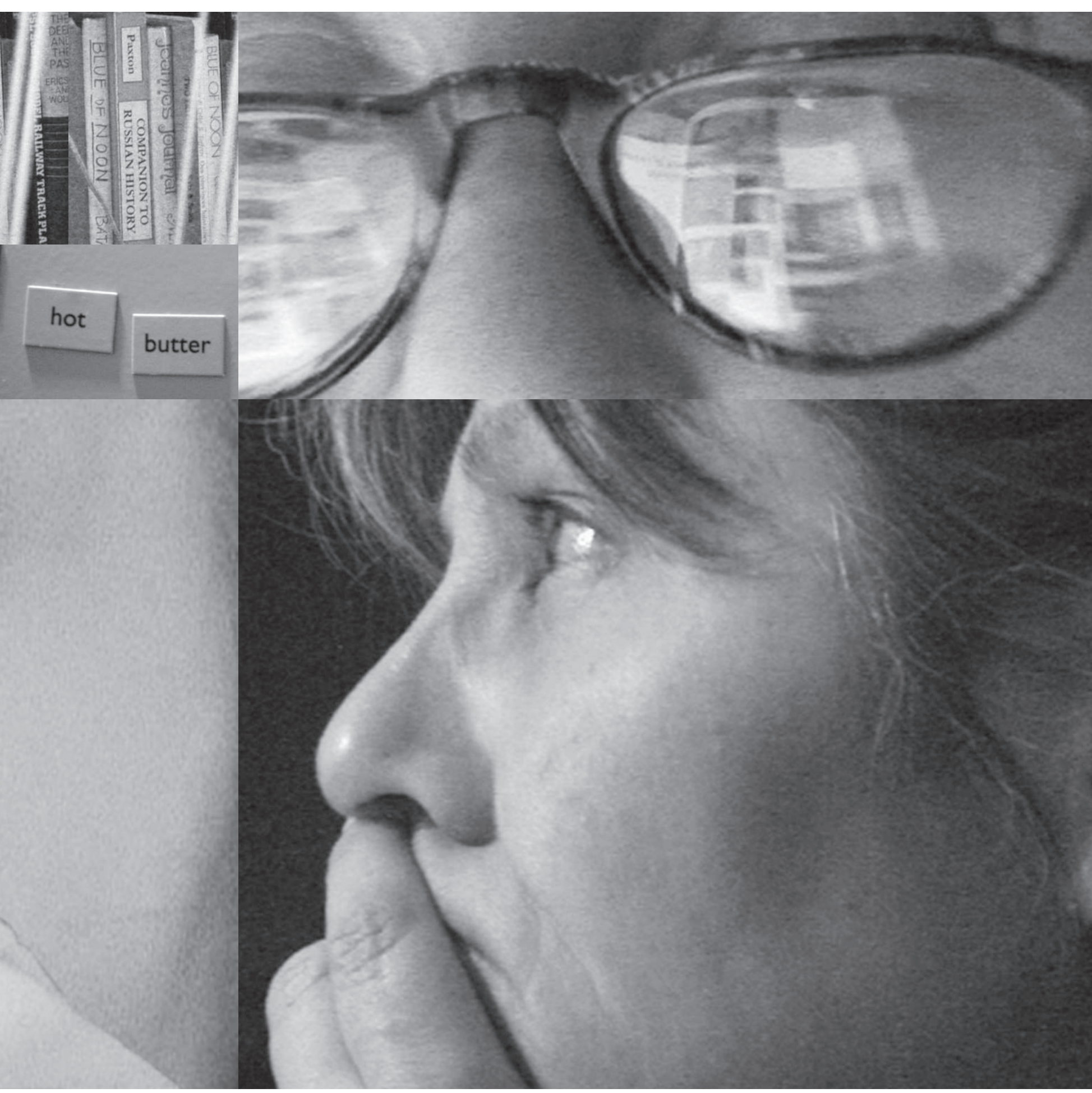




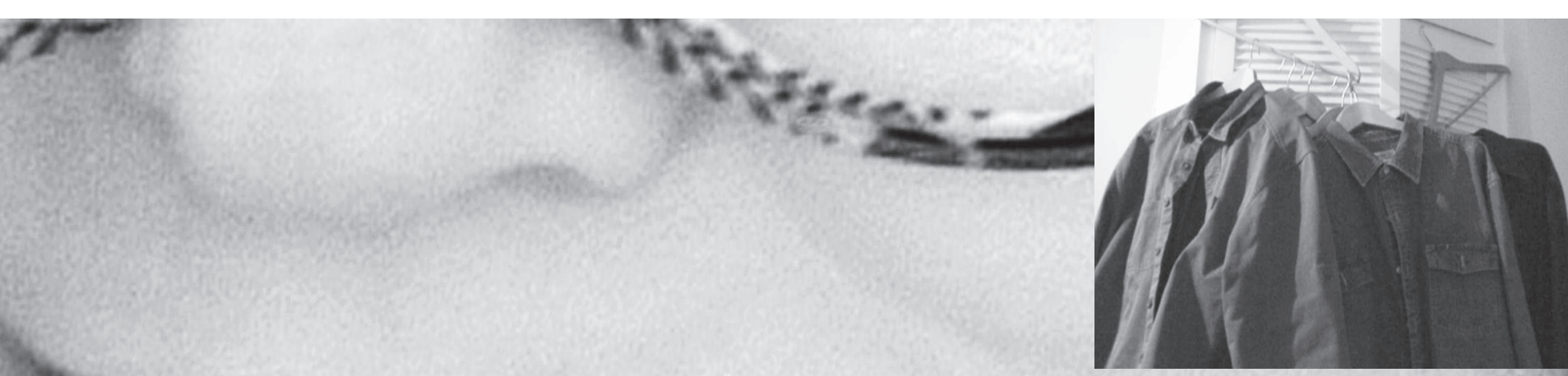

\section{4}

love

morning

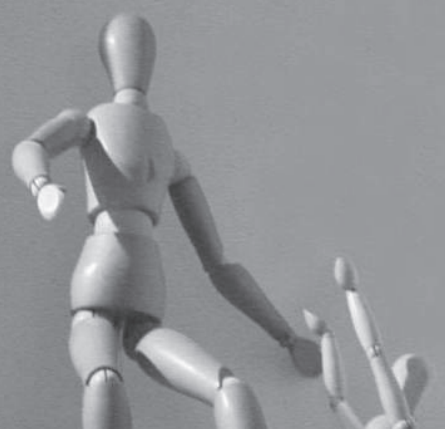

